\title{
Spontaneous chordoma: a case report on a female UM-HET3 mouse from the SLAM study
}

\author{
Eleonora Duregon ${ }^{a}$, Camila Vieira Ligo Teixeira ${ }^{\mathrm{a}}$, Dushani L. Palliyaguru ${ }^{\mathrm{a}}$, Annamaria L. Rudderow ${ }^{\mathrm{a}}$, \\ Victoria Hoffmann ${ }^{b}$, Michel Bernier ${ }^{a}$, Nathan L. Price ${ }^{a}$, Simonetta Camandola ${ }^{a}$, Rafael de Cabo ${ }^{a,}{ }^{*}$ \\ ${ }^{a}$ Translational Gerontology Branch, National Institute on Aging, National Institutes of Health, Baltimore, MD 21224, USA. \\ ${ }^{b}$ Division of Veterinary Resources, National Institutes of Health, Bethesda, MD 20892, USA.
}

\begin{abstract}
A female UM-HET3 mouse from the Study of Longitudinal Aging in Mice (SLAM) was euthanized at 164 weeks of age due to hind limb weakness. Necropsy and histological analysis revealed that the most probable cause of the clinical finding was the compression of the thoracolumbar segment of the spinal cord by herniated intervertebral disks. In addition, a spontaneous chordoma was incidentally found in the coccygeal bones. Given the rarity of this type of tumor, bio-clinical annotations acquired throughout lifespan, detailed histopathological assessment, and comparative clinical-pathological correlations for this mouse are presented and discussed.

Keywords: Geropathology, geroscience, study of longitudinal aging in mice (SLAM), spontaneous neoplasm, tumor burden, chordoma
\end{abstract}

\section{Introduction}

The Study of Longitudinal Aging in Mice (SLAM) is a large mouse population study instituted within the Intramural Research Program at the National Institute on Aging (NIA/NIH) [1] to comprehensively characterize normal aging in two commonly used strains of mice and to validate their usefulness as preclinical models for human aging and age-related diseases. Here, we report the case of a particularly long-lived SLAM female mouse in which a spontaneous chordoma was incidentally found during the necropsy.

\section{Case Report}

A 164-week-old female UM-HET3 mouse was humanely euthanized via carbon dioxide asphyxiation following veterinarian advice due to persistent hind limb weakness. The body was formalin-fixed and a complete necropsy was performed by the Division of Veterinary Resources (DVR/

\footnotetext{
* Corresponding author: Rafael de Cabo

Mailing address: Translational Gerontology Branch, National Institute on Aging, National Institutes of Health, Baltimore, MD 21224, USA.

Email: decaboRa@mail.nih.gov
}

Received: 01 December 2020 / Accepted: 16 December 2020
NIH, Bethesda, MD). The mouse was very lean, with muscle mass adequate for an aged mouse. Macroscopically, the liver was diffusely pale, as were the kidneys which also appeared to be smaller than normal. The major organs, gastro-intestinal tract, brain, and spinal cord were saved for histologic examination. Whole slide images of hematoxylin and eosin (H\&E)-stained tissue sections were acquired with a high-resolution Zeiss Axio Scan Z1 digital slide scanner (Zeiss, Oberkochen, Germany), and analyzed by a pathologist.

Microscopically, diffuse mild to moderate amyloid deposits were present in the heart (Figure 1A), liver (Figure 1B), spleen, small intestines (Figure 2B), mesentery, and subcutaneous fat. Both kidneys had moderate diffuse expansion of the glomerular mesangium, with pale eosinophilic deposits also present within glomerular capillary tufts. In addition, the right kidney had a severe tubular atrophy with $>90 \%$ of the nephrons being affected (Figure 1C). The lungs displayed a mild, multifocal acidophil macrophage Epneumonia (Figure 1D). In the duodenum, a pedunculated polyp projecting into the lumen was observed (Figure 2A), with mucosal cells forming dysplastic crypts (Figure 2B). Liver and spleen had aggregates of macrophages with pale gray granular pigment and scant dark brown polarizable granules. The brain had prominent gliosis and rare spheroids in large white matter tracts of the cerebrum. Bilateral symmetrical foci of gitter cells were present in the rostral ventrolateral aspect of the hippocampus. The deep cerebellar nuclei had rare swollen axons. Spinal nerve roots had dilated myelin sheaths and 
myelomacrophages. Disks of the thoracolumbar segment of the vertebral column were herniated and severely compressed the spinal cord. Thoracic spinal cord cranial to this compressed section had multifocal spheroids. Vertebrae and long bones showed thinning of cortical bone and widened lacunae with foci of replacement of bone marrow by new woven bone and fibrovascular connective tissue were present. Hematopoietic cells of the bone marrow had an increased proportion of plasma cells, some of which with large, atypical nuclei, suggesting a multiple myeloma. Finally, coccygeal bones displayed marrow cavities with normal fat replaced by sheets of markedly vacuolated polygonal cells (i.e. physaliferous cells) with small, round randomly located hyperchromatic nuclei (Figure 2C, D). Mitotic figures were not seen. This finding is consistent with an intramedullary chordoma, a very rare tumor of embryonic intervertebral disk cells. While the most probable cause of hindlimb weakness was the spinal cord compression, the reasons for symmetrical encephalomalacia and gliosis remain uncertain.

\section{Discussion}

SLAM was conceived as the mouse counterpart of the NIA Baltimore Longitudinal Study of Aging (BLSA), one of the world's oldest and still ongoing studies on healthy normative aging in humans [2]. Similar to BLSA, SLAM aims at defining individual mouse chronological normative aging, which is surprisingly lacking despite mice having been used for decades as a model of human aging, and to test preclinically lifespan extending compounds. Currently, most of the proposed metrics of aging stem from cross-sectional studies and rely on associations with chronological age that often fail to accurately predict mortality. Together with this, variables such as sex and age at administration of a given intervention have hindered the effectiveness of anti-aging strategies in mice and their translational applicability into humans [3]; hence the Geroscience research calls for a true model of functional or phenotypic normative mouse aging. Two strains of mice - the inbred C57BL/6J and outbred HET3 - of both sexes are employed in the SLAM study to account for genetic heterogeneity, sex- and strain-specific aging phenotypes, pathologies, and mortality patterns. To date, 2800 mice (700 mice per sex and strain, logistically separated into 14 cohorts of 200 mice) have been enrolled into the study, longitudinally evaluated for functional, phenotypic and biological health, and for collection of biospecimens throughout their lifespan and post-mortem. Within the longitudinal cohorts, a cross-sectional arm of the study has also been implemented to collect tissues and to generate a vast biorepository of serum, urine and feces, as well as flash frozen and formalin-fixed tissues [1].

The mouse of the present case report (SLAM794) belonged to Cohort 7 and was group-housed in a cage of four animals. They were ad libitum fed the open formula mouse diet, NIH-31 (3.0 kcal/g) (Envigo), with free access to water. The body weight of SLAM794 peaked (41 g) at 78 weeks, while still housed with two other cagemates (Figure 3A). The subsequent progressive decline of body weight was synchronous to the presence of only one

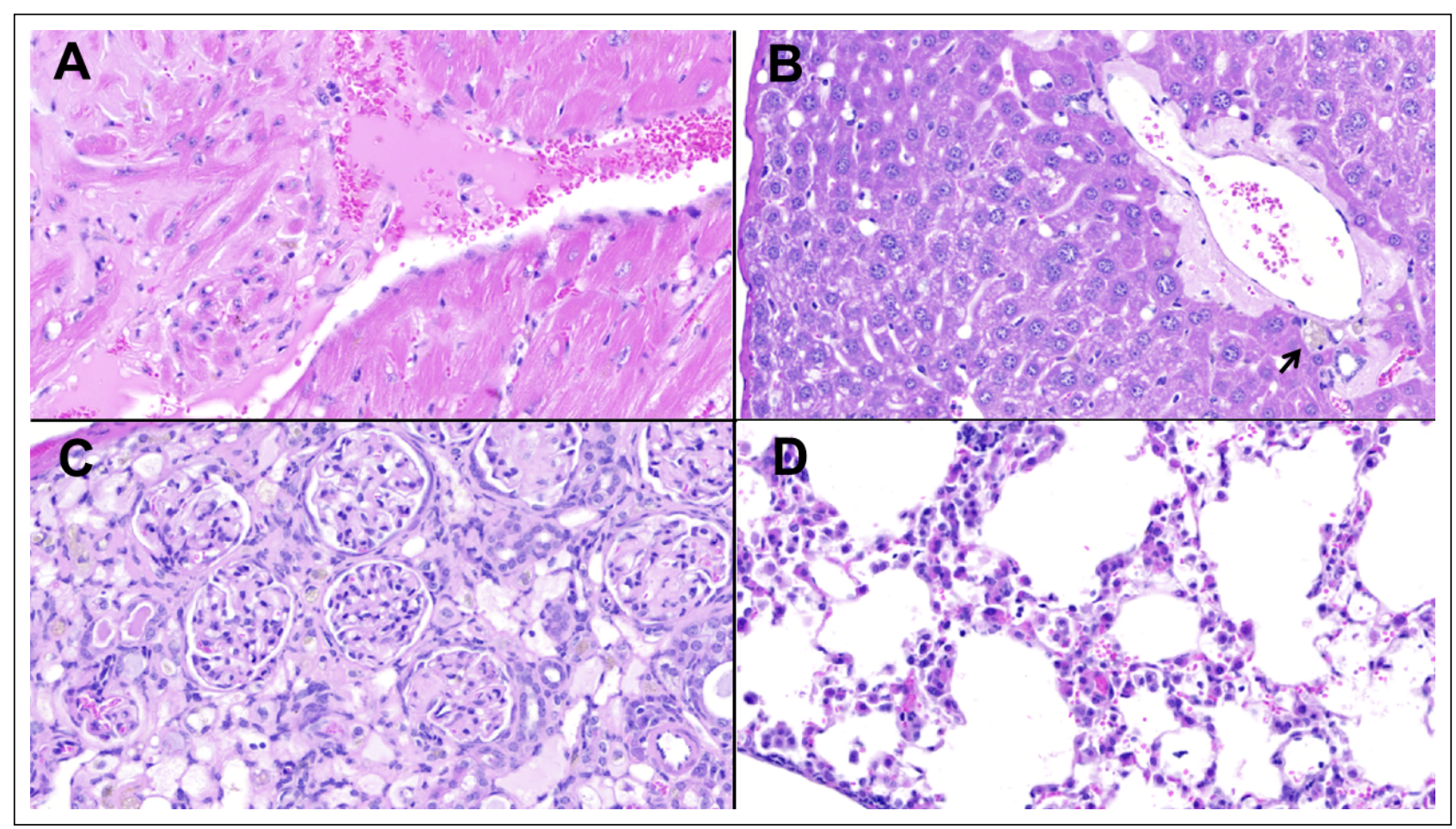

Figure 1. Selected non-neoplastic lesions. (A) Moderate amyloid deposits separating and replacing myocardial cells in the left ventricle, at the base of a papillary muscle. (B) Moderate amyloid deposits surrounding a portal region in the liver (arrow pointing at macrophages scant with dark brown polarizable granules). (C) Right kidney cortex showing a segmental, diffuse expansion of the glomerular mesangium and severe diffuse tubular atrophy. (D) Lungs displaying a mild, acidophil macrophage pneumonia. Hematoxylin and Eosin, original magnification 400x. 


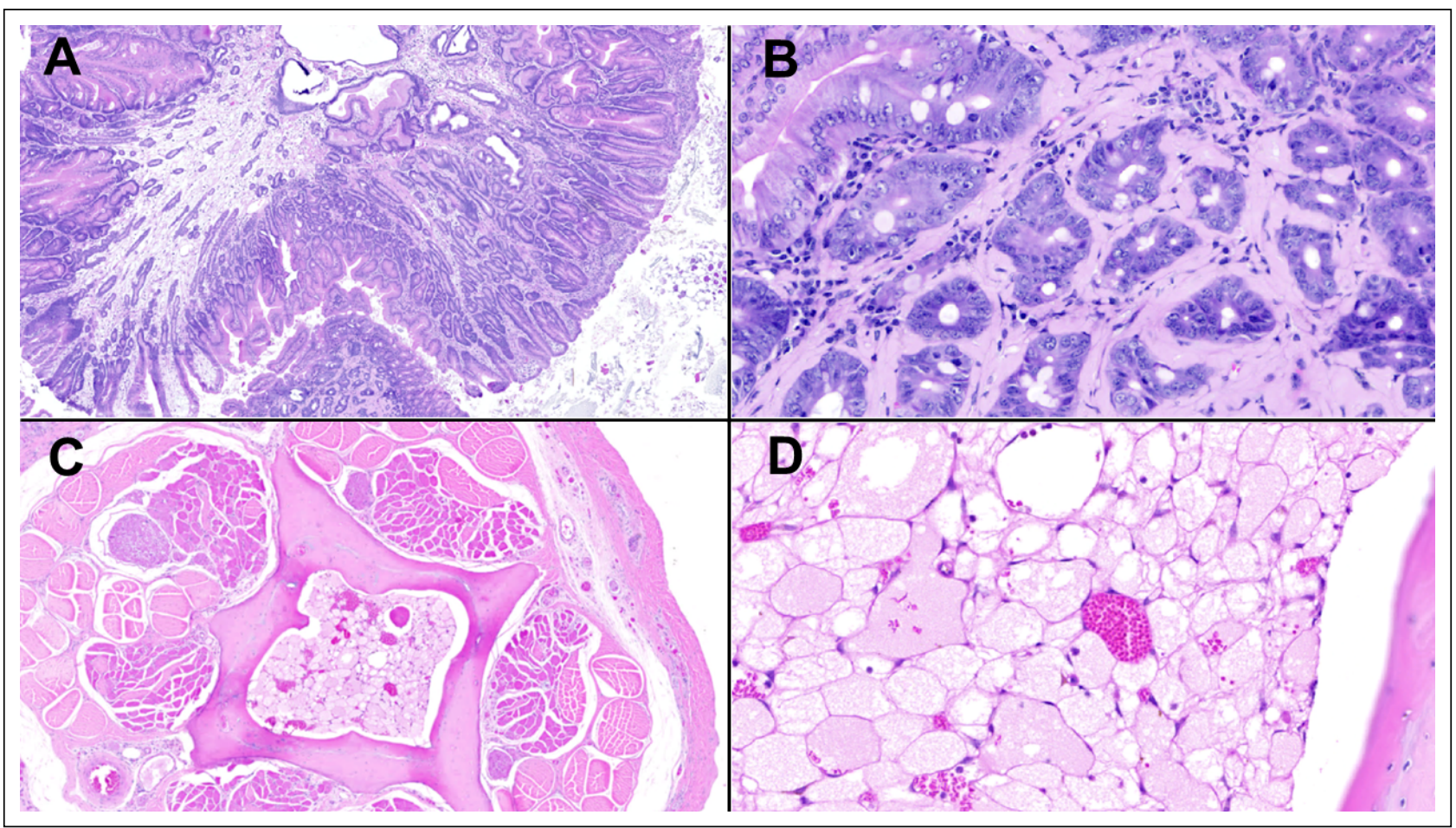

Figure 2. Selected neoplastic lesions. (A) Pedunculated duodenal polyp projecting into the lumen. (B) Amyloid deposits surrounding the stalk of the polyp dysplastic crypts. (C) Coccygeal bones marrow cavities replaced by an intramedullary chordoma, which is pathognomonically featured by markedly vacuolated polygonal cells (i.e. physaliferous cells) with small, round randomly located hyperchromatic nuclei. (D) Hematoxylin and Eosin, original magnification 40x in $\mathbf{A}, \mathbf{C}$; and 400x in B, D.

cagemate and then being single-housed. Motor function tests, including cage top and rotarod [4], were performed at 24-week intervals starting from 16 weeks of age. Both tests showed a reduction in the latency time, with the steepest decline from 16 to 40 weeks for cage top assessment and from 40 to 64 weeks for the rotarod test (Figure 3B). As expected, frailty index scores [4], assessed three times starting from 53 weeks, increased as the mouse (SLAM794) aged.

The examination of individual frailty index features that contributed to this increase showed that loss of whiskers and loss of vision occurred early and became permanent over time. Some impairment of forelimb grip strength was seen from 98 weeks onward. Kyphosis, hearing loss, tail stiffening, menace reflex impairment, and moderately high mouse grimace scale scores were observed at 132 weeks. The comparison of survival of this mouse to the full SLAM cohort showed that $99.7 \%$ of all mice were dead by the time it was euthanized at 164 weeks. In addition, more than $98.8 \%$ of HET3 female mice were dead at this time point. Upon necropsy and histopathological analyses, the disease burden, defined as the amount of histologic disease present at end of life in an individual [5], paralleled well with the clinical findings and was quite typical for a very long-lived mouse. As a matter of fact, instead of a highly lethal malignant tumor, such as lympho-reticular tumors, fibrosarcoma, and mammary adenocarcinoma, which would have killed the mouse at a younger age, a collection of slowly developing degenerative diseases (i.e. multiorgan amyloidosis, osteoporosis, encephalomalacia, and gliosis) and slowly-growing localized neoplastic diseases (i.e. duodenal dysplastic polyp, coccygeal chordoma, spinal multiple myeloma, for a total tumor burden of 3) was observed. In this regard, the spine alteration detected while assessing the frailty index was an early sign of the osteoporotic process that culminated in the disc herniation/spinal cord compression and chronic limb weakness requiring euthanasia. Along the same line, the intestinal and liver amyloidosis very likely contributed to the progressive weight loss.

Focusing on neoplasms, the coccygeal chordoma was a surprising incidental finding, being predominantly composed of pathognomonic physaliferous cells comparable to the typical form of chordomas described in humans. The lack of mitotic figures and morphological signs of invasion of the surrounding tissues supported a biologically indolent behavior. Chordomas are rare bone malignant neoplasms believed to be derived from notochord remnants that may persist anywhere along with the axial skeleton [6]. In humans, they can be sporadic or familial and account for $1-4 \%$ of all bone malignancies, with an incidence of 0.08 per 100,000 in the United States [7]. They are generally slow growing, locally invasive, chemotherapy-resistant and radiotherapy-resistant tumors. However, due to their location, chordomas are a highly morbid and potentially fatal disease that requires complex medical decisions and tailored patient care. Despite having been reported in many vertebrate species, including fish and numerous mammals (i.e. dog, cat, ferret, mink, Sprague-Dawley and Fischer 344 rat), epidemiological 


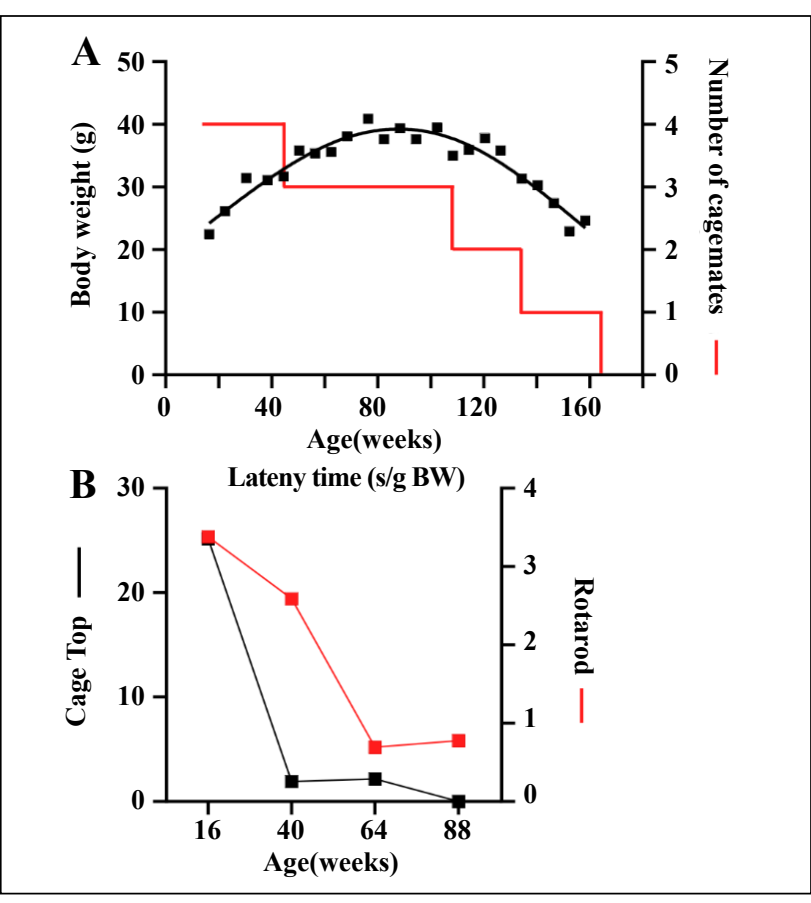

Figure 3. Longitudinal and cross-sectional measurements assessed for mouse SLAM794. (A) Bodyweight trajectory and housing density over the mouse lifespan. (B) Motor function tests were performed at 24 week intervals starting from 16 weeks of age: Cage top (black symbols) and rotarod (red symbols).

data are scarce due to their rarity. Recently, an interesting high prevalence of chordomas was reported among the captive population of the endangered Perdido Key Beach Mouse (PKB mouse, Peromyscus polionotus trissyllepsis) [8]. With 38 out of 88 mice $(43 \%)$ bearing a chordoma, without any significant sexual dimorphism differences, a genetic predisposition was the most likely explanation for this unusually high prevalence, especially when considering the known bottlenecks and inbreeding that have occurred in these captive PKB mice. In humans, both the sporadic and the familial forms of chordomas have been linked to the $t b x t$ gene (6q27), which encodes the Tbox transcription factor $\mathrm{T}$ or brachyury homologue. This protein is important in the development of the notochord and is highly expressed in chordoma cells. Duplication of the tbxt gene has been shown to be a predisposing factor for familial chordoma [9]. It is thus possible that PKB mice carry a similar or alternative germline mutation, that, coupled with founder effect, predisposes the captive population to chordoma and ensures it is the prevalent cause of mortality as they age (chordoma prevalence was $50 \%$ and $71 \%$ in mice 4 and 5 years old) [8]. On the other hand, the chordoma described in the present report appears more similar to the human sporadic form of chordoma as it was found in a heterogeneous mouse strain,
HET3, that typically has a varied spectrum of age-related mortality causes. As the collection and in-depth analysis of data generated by SLAM move forward, it will be very interesting to see how rarely reported pathologies, like the chordoma discussed here, will cluster in relation to strain, sex, age, and subsequent clinicopathological correlations, providing a strong foundation for translational research.

\section{Declarations}

Acknowledgments: This research was supported by the Intramural Research Program of the National Institute on Aging of the National Institutes of Health.

Conflict of interest: The authors declare that they have no conflict of interest.

\section{References}

1. Palliyaguru D L, Vieira Ligo Teixeira C, Duregon E, et al. Study of Longitudinal Aging in Mice: Presentation of Experimental Techniques (SLAM POET). The Journals of Gerontology: Series A, 2020.

2. Shock N W. A New Research Building for Gerontology. The Gerontologist, 1964, 4(4): 185-189.

3. Gonzalez-Freire M, Diaz-Ruiz A, Hauser D, et al. The road ahead for health and lifespan interventions. Ageing Research Reviews, 2020: 101037.

4. Bellantuono I, de Cabo R, Ehninger D, et al. A toolbox for the longitudinal assessment of healthspan in aging mice. Nature protocols, 2020, 15(2): 540-574.

5. Snyder J M, Ward J M, Treuting P M. Cause-of-death analysis in rodent aging studies. Veterinary pathology, 2016, 53(2): 233-243.

6. Walcott B P, Nahed B V, Mohyeldin A, et al. Chordoma: current concepts, management, and future directions. The lancet oncology, 2012, 13(2): e69-e76.

7. McMaster M L, Goldstein A M, Bromley C M, et al. Chordoma: incidence and survival patterns in the United States, 1973-1995. Cancer Causes \& Control, 2001, 12(1): 1-11.

8. Taylor K R, Garner M M, Russell K, et al. Chordomas at high prevalence in the captive population of the endangered Perdido Key beach mouse (Peromyscus polionotus trissyllepsis). Veterinary pathology, 2016, 53(1): 163169.

9. Yang X R, Ng D, Alcorta D A, et al. T (brachyury) gene duplication confers major susceptibility to familial chordoma. Nature genetics, 2009, 41(11): 1176-1178.

Cite this article as: Duregon E, Teixeira C V L, Palliyaguru D L, et al. Spontaneous chordoma: a case report on a female UM-HET3 mouse from the SLAM study[J]. Aging Pathobiology and Therapeutics, 2020, 2(4): 219222. 